\title{
Proposed Mechanisms of Photobiomodulation (PBM) Mediated via the Stimulation of Mitochondrial Activity in Peripheral Nerve Injuries
}

\author{
Ji Eun Choi ${ }^{1,2}$
}

'Department of Otorhinolaryngology-Head and
Neck Surgery, Dankook University Hospital,
Cheonan, Korea
${ }^{2}$ Department of Medical Science, College of
Medicine, Dankook Univeristy, Cheonan, Korea

Received August 2, 2021

Accepted September 24, 2021

\section{Correspondence}

Ji Eun Choi

Department of Otorhinolaryngology-Head and Neck Surgery, Dankook University College of Medicine, 119 Dandae-ro, Dongnam-gu,

Cheonan 31116, Korea

Tel.: +82-41-550-3284

Fax: $+82-41-556-1090$

E-mail: garimungdamail.com

(C) Korean Society for Laser Medicine and Surgery

(c) This is an open access article distributed under the terms of the Creative Commons Attribution NonCommercial License (http://creativecommons.org/ licenses/by-nc/4.0) which permits unrestricted noncommercial use, distribution, and reproduction in any medium, provided the original work is properly cited.
Evidence shows that nerve injury triggers mitochondrial dysfunction during axonal degeneration. Mitochondria play a pivotal role in axonal regeneration. Therefore, normalizing mitochondrial energy metabolism may represent an elective therapeutic strategy contributing to nerve recovery after damage. Photobiomodulation (PBM) induces a photobiological effect by stimulating mitochondrial activity. An increasing body of evidence demonstrates that PBM improves ATP generation and modulates many of the secondary mediators [reactive oxygen species (ROS), nitric oxide (NO), cyclic adenosine monophosphate (cAMP), and calcium ions $\left.\left(\mathrm{Ca}^{2+}\right)\right]$, which in turn activate multiple pathways involved in axonal regeneration.

\section{Key words}

Axon regeneration; Mitochondria; Photobioodulation; Peripheral nervous system 


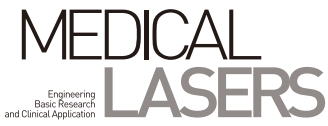

\section{INTRODUCTION}

Mitochondria are dynamic organelles playing a pivotal role in energy generation, signal transmission, and calcium homeostasis essential for neurons due to their high energy consumption. ${ }^{1}$ Most axonal mitochondria are stationary and exhibit a linearly interspersed distribution in stationary state. ${ }^{2}$ Axonal regeneration in the peripheral nervous system (PNS) initiates a vigorous regenerative response via permissive Schwann cell environment and results in the formation of a new growth cone. During axonal regeneration, mitochondria are transported to the proximal segment of injured axons, which require large energy for regeneration. ${ }^{3.4}$ This process consumes a large amount of energy and molecules, and therefore altered mitochondrial behavior may represent one of the key intrinsic mechanisms for peripheral nerve regeneration.

Photobiomodulation (PBM) entails absorption of nonionizing optical radiation in the visible and near-infrared spectral range by endogenous chromophores to elicit photo-physical and photo-chemical events at various biological scales. ${ }^{5,6}$ Mitochondria contain chromophores for the absorption of photons during PBM. In this review, we summarize the behavior of mitochondria and elucidate their role in axonal degeneration following peripheral nerve injury. We also describe the therapeutic mechanism of PBM based on these effects.

\section{MITOCHONDRIAL BEHAVIOR FOLLOWING PERIPHERAL NERVE INJURY}

In contrast to the central nervous system, the peripheral nerve system exhibits a high regenerative capacity. Injury to the peripheral nerve triggers Wallerian degeneration of the distal axon to create a microenvironment conducive for axonal regrowth and reinnervation, while the proximal axons regenerate growth cones that drive axon elongation.

Axonal degeneration is intimately associated with mitochondrial dysfunction, including decreased adenosine triphosphate (ATP) synthesis, increased generation of reactive oxygen species (ROS), disequilibrium of mitochondrial fission and fusion, impaired axonal mitochondrial transport, and aberrant mitophagy. ${ }^{8-12}$ Under normal physiological conditions in neurons, the principal role of mitochondria is ATP production via oxidative phosphorylation using the respiratory machinery, composed of four respiratory complexes and the F1FO ATP synthase complexes. ${ }^{13}$ The energy needs of the nervous system are enormous, primarily to restore the ionic balance after the generation and transmission of nerve impulses through the activity of the $\mathrm{Na}^{+} / \mathrm{K}^{+}$ATPase, an ATP-dependent pump. ${ }^{14}$ Several studies reported that nerve injuries increase the oxygen consumption without increasing ATP production. ${ }^{15}$ This ATP dissipation phenomenon may depend on mitochondrial uncoupling lany pathway that enables proton re-entry into the matrix independent of ATP production) or F-ATP synthase uncoupling lany condition that inhibits the coupling between the F1 catalytic activities and the proton translocation by F0). ${ }^{16,17}$ Thus, the reduced levels of ATP following nerve injury can reduce the $\mathrm{Na}^{+} / \mathrm{K}^{+}$ATPase activity, and interfere with restoration of the ionic equilibrium by neurons. Mitochondria also play a role in the production and detoxification of ROS. ${ }^{18-20}$ Mitochondrial dysfunction leads to increased ROS either by the organelle itself or indirectly by the endoplasmic reticulum (ER). ${ }^{20,21}$ This oxidative stress is exacerbated by the inflammation associated with neuronal damage, creating a vicious circle. ${ }^{22}$ Oxidative stress damages the inner mitochondrial membrane housing oxidative phosphorylation (OXPHOS) machinery, ${ }^{23}$ which in turn reduces aerobic ATP production, and thereby induces a metabolic switch to anaerobic glycolysis to restore the intracellular levels of ATP. ${ }^{15,24}$ High ROS levels trigger a vicious circle of ROS-stimulated glucose uptake and glucose-stimulated ROS production. ${ }^{24}$ Since mitochondria play a pivotal role, together with the ER, in the maintenance of cytosolic $\mathrm{Ca}^{2+}$ levels, ${ }^{25,26}$ the altered mitochondrial metabolism leads to cytosolic $\mathrm{Ca}^{2+}$ imbalance.

Several studies reported that multiple proteins are inhibited or activated in response to injury during axonal degeneration. Active degeneration requires Sterile alpha and TIR motif containing 1 (SARM1) and mitogen-activated protein kinase (MAPK) including dual leucine zipper kinase (DLK), while the nicotinamide mononucleotide adenylyl transferase (NMNAT) attenuates Wallerian degeneration. NMNAT, a key enzyme mediating nicotinamide adenine dinucleotide (NAD+) synthesis, is well known for its neuroprotective function mediated via modification of mitochondrial function. ${ }^{27-30}$ The activity of endogenous NMNAT is greatly inhibited by defective mitochondrial structure and function, which decreases mitochondrial transport, and ROS accumulation following axonal injury. ${ }^{27,31-33}$ Together with increased intra-axonal $\mathrm{Ca}^{2+}$ levels, the rapid decrease in NAD-biosynthetic enzyme NMNAT activates SARM1, which triggers Wallerian degeneration. ${ }^{34-38}$ SARM1 triggers NAD+ depletion and activates mitogen activated protein kinase (MAPK) signaling. ${ }^{39,40}$ The DLK (or MAP3K), an axonal integrity sensor, interacts with NMNAT and activated SARM1. 36,37,41,42 SARM1- 
MAPK pathway disrupts axonal energy homeostasis by reducing ATP production before physical breakdown of damaged axons. ${ }^{36}$ Decreased NAD+ synthesis reduces energy generation and ATP levels, causing defects in $\mathrm{Na}^{+} / \mathrm{Ca}^{2+}$ exchangers and $\mathrm{Ca} 2+$ channels, and the loss of mitochondrial membrane potential (MMP). ${ }^{39,43}$ Thus collectively, these proteins damage axonal integrity.

Mitochondria are essential for axon regeneration.Increased mitochondrial density effectively promotes axonal regeneration. ${ }^{1,44}$ Increasing mitochondrial density at the proximal segment of injured axons is achieved, mainly by increasing mitochondrial transport and mitochondrial fission. In vivo studies indicate that enhanced mitochondrial transport and early mitochondrial fission after axon damage accelerates axonal regeneration. ${ }^{1,45,46}$ Mitochondria not only supply the structural components of cells during axonal transport, but also provide appropriate levels of ATP to the growth cone of regenerating axons. ${ }^{47,48}$ In an in vitro study of sciatic nerve injury, the axon cyclin-dependent kinase 5 (CDK5) levels were increased after nerve injury and translocated into the mitochondrial membrane of regenerating axons. ${ }^{49}$ Mitochondrial CDK5 phosphorylates mitochondrial signal transducer and activator of transcription 3 (STAT3), thereby increasing mitochondrial membrane potential. By interacting with electron transport chain proteins, STAT3 regulates ROS production and cytochrome c release, and contributes to ATP energy supply for axonal regeneration. ${ }^{50}$

Therefore, approaches to stimulate mitochondrial activity may provide an effective therapeutic strategy to enhance axonal regeneration in the injured peripheral nerve.

\section{PBM STIMULATES MITOCHONDRIAL ACTIVITY}

Mitochondria in the injured axon play a pivotal role in axonal degeneration and regeneration. Increased mitochondrial transport facilitates the replacement of damaged mitochondria by healthy ones to provide adequate ATP energy for axonal regeneration. PBM exerts a photobiological effect via stimulation of cytochrome c oxidase (CCO) located in the cytochrome of the mitochondrial respiratory chain complex IV. It has been hypothesized that PBM enhances the activity of $\mathrm{CCO}$ and subsequently improves the generation of ATP. ${ }^{51}$ Pho-dissociation of nitric oxide (NO) from CCO leads to the gain of an electron from each of the four cytochrome $\mathrm{C}$ molecules by $\mathrm{CCO}$, which then transfers four protons to a single oxygen to form two molecules of water. This process contributes to the formation of the proton gradient, which drives the activity of ATP synthase. CCO activation by PBM enhances the synthesis of ATP and consequent modulation of ROS production, $\mathrm{NO}$ release and $\mathrm{Ca}^{2+}$ homeostasis. ${ }^{52-54}$

Extracellular ATP induces $\mathrm{Ca}^{2+}$ influx by binding to PX2 receptors, which are membrane ion channels selectively permeable to $\mathrm{Na}^{+}, \mathrm{K}^{+}$and $\mathrm{Ca}^{2+}$. Binding of the ATP to $P 2 Y$ receptors, which are G-protein-coupled receptors, triggers the release of $\mathrm{Ca}^{2+}$ from the ER via inositol 1,4,5-trisphosphate (IP3) and diacylglycerol (DAG) pathway. ${ }^{55}$ Thus, the increased ATP synthesis due to CCO stimulation by PBM induces $\mathrm{Ca}^{2+}$ influx together with the ER. Also, PBM increases the level of cyclic adenosine monophosphate (cAMP) synthesized from ATP by adenylate cyclase. . $^{5,57}$

PBM is known to activate multiple pathways such as MAPK/extracellular signal-regulated kinase (ERK) pathway, or phosphatidylinositol 3-kinase (PI3K)/protein kinase B (AKT) pathway, ${ }^{58,59}$ in various molecular networks in the cellular system. $\mathrm{Ca}^{2+}$ influx and increase in intracellular $\mathrm{Ca}^{2+}$ activate MAPK/ERK or PI3K/AKT via calmodulin. ${ }^{60}$ The second messenger CAMP activates MAPK/ERK pathways ${ }^{61}$ and ROS signaling leads to the activation of PI3K/AKT pathway. ${ }^{62}$ ERK/MAPK and PI3K/AKT signal channels are known to regulate cell survival, growth, and proliferation, and possibly play an important role in regeneration after nerve injury. ${ }^{63-65}$ Genetic elevation of $\mathrm{Ca}^{2+}$ or cAMP accelerates the formation of a growth cone from the injured axon, facilitates apparent fusion of axonal fragments, and promotes branching to postsynaptic targets. ${ }^{66}$ Thus, PBM stimulates mitochondrial activity, and subsequently promotes regeneration of peripheral nerve after injury. For example, studies reported that PBM promotes axonal regeneration of crushed facial nerves by u-regulating the PI3K/AKT signaling pathways. ${ }^{59}$ and restores $\mathrm{Na}$, K-ATPase disrupted in response to ouabain via MAPK/ERK pathway. ${ }^{67}$ These findings suggest that PBM directly or indirectly supports injured nerve recovery via mitochondrial bioenergetics. Nevertheless, further studies are still needed to elucidate the mechanism of PBM in axonal degeneration and regeneration by stimulating mitochondrial activity.

\section{DISCUSSION}

Understanding the mechanisms regulating axonal degeneration and regeneration is crucial for developing treatments for nerve injury. Mitochondrial dysfunction in the injured axon plays a pivotal role in initiation of axonal degeneration and inhibition of axonal regeneration. ${ }^{8-12}$ Thus, the present knowledge of mitochondrial dysfunc- 
tion following peripheral nerve injury may facilitate therapeutic interventions to aid axonal regeneration. Despite the elusive underlying mechanism, mitochondrial dysfunction not only causes ATP production and oxidative stress but also activates multiple proteins that damage axonal integrity. ${ }^{27-32}$ In contrast, enhancing mitochondrial function is an important intrinsic mechanism of axonal regeneration. ${ }^{1,44-46}$ Accumulating evidence demonstrates that axonal regeneration requires adequate energy production and mitochondrial transport to the growth cone of regenerating axons. Therefore, treatment strategies that directly target mitochondria will enable axonal regeneration by mitigating local ROS production or restoring $\mathrm{Ca}^{2+}$ homeostasis.

In the last decade, PBM has been introduced as a noninvasive treatment to enhance the recovery from peripheral nerve injury based on the leading hypothesis of the mechanism that the photons dissociate inhibitory NO from the CCO, resulting in increased electron transport, MMP and ATP production. Stimulation of mitochondrial activity activates numerous signaling pathways via ROS, CAMP, NO and $\mathrm{Ca}^{2+}$, leading to the activation of MAPK/ ERK or PI3K/AKT pathways. ${ }^{46,52,53,63}$ These signaling pathways lead to axonal regeneration. Despite the effectiveness of the PBM mechanism in mitochondria, several molecules and substrates participate in the mitochondrial dynamics to facilitate axonal regeneration. Further studies are needed to fill the gap between axonal regeneration and mechanisms underlying PBM. Further, the switch to human studies is not easy, due to the complexity of different wavelengths with unpredictable depth of penetration to the injured nerve through tissues of different type and thickness.

\section{CONFLICT OF INTEREST}

Ji Eun Choi is an editorial board member of the journal but was not involved in the review process of this manuscript. Otherwise, there is no conflict of interest to declare.

\section{FUNDING}

This work was supported by a National Research Foundation of Korea (NRF) grant funded by the Korean government (MSIT) (no. NRF-2020R1C1C1009849).

\section{ORCID}

Ji Eun Choi, https://orcid.org/0000-0001-8105-813X

\section{REFERENCES}

1. Han SM, Baig HS, Hammarlund M. Mitochondria localize to injured axons to support regeneration. Neuron 2016;92:1308-23.

2. Cheng XT, Sheng ZH. Developmental regulation of microtubule-based trafficking and anchoring of axonal mitochondria in health and diseases. Dev Neurobiol 2021;81:284-99.

3. Morris RL, Hollenbeck PJ. The regulation of bidirectional mitochondrial transport is coordinated with axonal outgrowth. J Cell Sci 1993;104(Pt 3):917-27.

4. Tao K, Matsuki N, Koyama R. AMP-activated protein kinase mediates activity-dependent axon branching by recruiting mitochondria to axon. Dev Neurobiol 2014;74:557-73.

5. Ravera S, Colombo E, Pasquale C, Benedicenti S, Solimei L, Signore $\mathrm{A}$, et al. Mitochondrial bioenergetic, photobiomodulation and trigeminal branches nerve damage, what's the connection? A review. Int J Mol Sci 2021;22:4347.

6. Bathini M, Raghushaker CR, Mahato KK. The molecular mechanisms of action of photobiomodulation against neurodegenerative diseases: a systematic review. Cell Mol Neurobiol. In press 2020.

7. Neukomm LJ, Burdett TC, Seeds AM, Hampel S, CoutinhoBudd JC, Farley JE, et al. Axon death pathways converge on Axundead to promote functional and structural axon disassembly. Neuron 2017;95:78-91.e5.

8. Court FA, Coleman MP. Mitochondria as a central sensor for axonal degenerative stimuli. Trends Neurosci 2012;35:364-72.

9. Sheng ZH, Cai Q. Mitochondrial transport in neurons: impact on synaptic homeostasis and neurodegeneration. Nat Rev Neurosci 2012;13:77-93.

10. Geden MJ, Deshmukh M. Axon degeneration: context defines distinct pathways. Curr Opin Neurobiol 2016;39:108-15.

11. Lin MT, Beal MF. Mitochondrial dysfunction and oxidative stress in neurodegenerative diseases. Nature 2006;443:787-95.

12. Baloh RH, Schmidt RE, Pestronk A, Milbrandt J. Altered axonal mitochondrial transport in the pathogenesis of CharcotMarie-Tooth disease from mitofusin 2 mutations. J Neurosci 2007;27:422-30.

13. Lenaz G, Genova ML. Structure and organization of mitochondrial respiratory complexes: a new understanding of an old subject. Antioxid Redox Signal 2010;12:961-1008.

14. Pirahanchi Y, Jessu R, Aeddula NR. Physiology, sodium potassium pump. Treasure Island: StatPearls Publishing; 2021.

15. Lim TK, Rone MB, Lee S, Antel JP, Zhang J. Mitochondrial and bioenergetic dysfunction in trauma-induced painful peripheral neuropathy. Mol Pain 2015;11:58.

16. Demine S, Renard P, Arnould T. Mitochondrial uncoupling: a key controller of biological processes in physiology and diseases. Cells 2019;8:795.

17. Lippe G, Coluccino G, Zancani M, Baratta W, Crusiz P. Mito- 
chondrial F-ATP synthase and its transition into an energydissipating molecular machine. Oxid Med Cell Longev 2019;2019:8743257.

18. Murphy MP. How mitochondria produce reactive oxygen species. Biochem J 2009;417:1-13.

19. Giorgio M, Trinei M, Migliaccio E, Pelicci PG. Hydrogen peroxide: a metabolic by-product or a common mediator of ageing signals? Nat Rev Mol Cell Biol 2007;8:722-8.

20. Venditti P, Di Stefano L, Di Meo S. Mitochondrial metabolism of reactive oxygen species. Mitochondrion 2013;13:71-82.

21. Murphy MP. Mitochondrial dysfunction indirectly elevates ROS production by the endoplasmic reticulum. Cell Metab 2013;18:145-6.

22. Solleiro-Villavicencio H, Rivas-Arancibia S. Effect of chronic oxidative stress on neuroinflammatory response mediated by CD4+T cells in neurodegenerative diseases. Front Cell Neurosci 2018;12:114.

23. Tiwari BS, Belenghi B, Levine A. Oxidative stress increased respiration and generation of reactive oxygen species, resulting in ATP depletion, opening of mitochondrial permeability transition, and programmed cell death. Plant Physiol 2002;128:127181.

24. Liemburg-Apers DC, Willems PH, Koopman WJ, Grefte S. Interactions between mitochondrial reactive oxygen species and cellular glucose metabolism. Arch Toxicol 2015;89:1209-26.

25. Rizzuto R, De Stefani D, Raffaello A, Mammucari C. Mitochondria as sensors and regulators of calcium signalling. Nat Rev Mol Cell Biol 2012;13:566-78.

26. Lee S, Min KT. The interface between ER and mitochondria: molecular compositions and functions. Mol Cells 2018;41:10007.

27. O'Donnell KC, Vargas ME, Sagasti A. WIdS and PGC-1 $\alpha$ regulate mitochondrial transport and oxidation state after axonal injury. J Neurosci 2013;33:14778-90.

28. Wang Y, Zhao D, Pan B, Song Z, Shah SZA, Yin X, et al. Death receptor 6 and caspase- 6 regulate prion peptide-induced axonal degeneration in rat spinal neurons. J Mol Neurosci 2015;56:966-76.

29. Conforti L, Tarlton A, Mack TG, Mi W, Buckmaster EA, Wagner D, et al. A Ufd2/D4Cole1e chimeric protein and overexpression of Rbp7 in the slow Wallerian degeneration (WldS) mouse. Proc Natl Acad Sci U S A 2000;97:11377-82.

30. Press C, Milbrandt J. Nmnat delays axonal degeneration caused by mitochondrial and oxidative stress. J Neurosci 2008;28:4861-71.

31. Yahata N, Yuasa S, Araki T. Nicotinamide mononucleotide adenylyltransferase expression in mitochondrial matrix delays Wallerian degeneration. J Neurosci 2009;29:6276-84.

32. Avery MA, Rooney TM, Pandya JD, Wishart TM, Gillingwater TH, Geddes JW, et al. WIdS prevents axon degeneration through in- creased mitochondrial flux and enhanced mitochondrial Ca2+ buffering. Curr Biol 2012;22:596-600.

33. Fang $Y$, Soares L, Teng X, Geary M, Bonini NM. A novel Drosophila model of nerve injury reveals an essential role of Nmnat in maintaining axonal integrity. Curr Biol 2012;22:590-5.

34. Adalbert R, Morreale G, Paizs M, Conforti L, Walker SA, Roderick $\mathrm{HL}$, et al. Intra-axonal calcium changes after axotomy in wild-type and slow Wallerian degeneration axons. Neuroscience 2012;225:44-54.

35. Ma M, Ferguson TA, Schoch KM, Li J, Qian Y, Shofer FS, et al. Calpains mediate axonal cytoskeleton disintegration during Wallerian degeneration. Neurobiol Dis 2013;56:34-46.

36. Yang J, Wu Z, Renier N, Simon DJ, Uryu K, Park DS, et al. Pathological axonal death through a MAPK cascade that triggers a local energy deficit. Cell 2015;160:161-76.

37. Gerdts J, Summers DW, Milbrandt J, DiAntonio A. Axon selfdestruction: new links among SARM1, MAPKs, and NAD+ metabolism. Neuron 2016;89:449-60.

38. Osterloh JM, Yang J, Rooney TM, Fox AN, Adalbert R, Powell $E H$, et al. dSarm/Sarm1 is required for activation of an injuryinduced axon death pathway. Science 2012;337:481-4.

39. Gerdts J, Brace EJ, Sasaki Y, DiAntonio A, Milbrandt J. SARM1 activation triggers axon degeneration locally via $\mathrm{NAD}^{+}$destruction. Science 2015;348:453-7.

40. Wang J, Zhai Q, Chen Y, Lin E, Gu W, McBurney MW, et al. A local mechanism mediates NAD-dependent protection of axon degeneration. J Cell Biol 2005;170:349-55.

41. Cavalli V, Kujala P, Klumperman J, Goldstein LS. Sunday Driver links axonal transport to damage signaling. J Cell Biol 2005;168:775-87.

42. Xiong T, Tang J, Zhao J, Chen H, Zhao F, Li J, et al. Involvement of the Akt/GSK-3B/CRMP-2 pathway in axonal injury after hypoxic-ischemic brain damage in neonatal rat. Neuroscience 2012;216:123-32.

43. Loreto A, Di Stefano M, Gering M, Conforti L. Wallerian degeneration is executed by an NMN-SARM1-dependent late $\mathrm{Ca}^{2+}$ influx but only modestly influenced by mitochondria. Cell Rep 2015;13:2539-52.

44. Kuo CC, Su HL, Chang TL, Chiang CY, Sheu ML, Cheng FC, et al. Prevention of axonal degeneration by perineurium injection of mitochondria in a sciatic nerve crush injury model. Neurosurgery 2017;80:475-88.

45. Zhou B, Yu P, Lin MY, Sun T, Chen Y, Sheng ZH. Facilitation of axon regeneration by enhancing mitochondrial transport and rescuing energy deficits. J Cell Biol 2016;214:103-19.

46. Kiryu-Seo S, Tamada H, Kato Y, Yasuda K, Ishihara N, Nomura $M$, et al. Mitochondrial fission is an acute and adaptive response in injured motor neurons. Sci Rep 2016;6:28331.

47. Prior R, Van Helleputte L, Benoy V, Van Den Bosch L. Defective axonal transport: a common pathological mechanism in 
inherited and acquired peripheral neuropathies. Neurobiol Dis 2017;105:300-20.

48. Mahar M, Cavalli V. Intrinsic mechanisms of neuronal axon regeneration. Nat Rev Neurosci 2018;19:323-37.

49. Hwang J, Namgung U. Phosphorylation of STAT3 by axonal Cdk5 promotes axonal regeneration by modulating mitochondrial activity. Exp Neurol 2021;335:113511.

50. Szczepanek K, Chen Q, Derecka M, Salloum FN, Zhang Q, Szelag $M$, et al. Mitochondrial-targeted Signal transducer and activator of transcription 3 (STAT3) protects against ischemiainduced changes in the electron transport chain and the generation of reactive oxygen species. J Biol Chem 2011;286:2961020.

51. Hamblin MR. Mechanisms and mitochondrial redox signaling in photobiomodulation. Photochem Photobiol 2018;94:199-212.

52. Hamblin MR. Shining light on the head: photobiomodulation for brain disorders. BBA Clin 2016;6:113-24.

53. Wong-Riley MT, Liang HL, Eells JT, Chance B, Henry MM, Buchmann E, et al. Photobiomodulation directly benefits primary neurons functionally inactivated by toxins: role of cytochrome c oxidase. J Biol Chem 2005;280:4761-71.

54. Lee JH, Carpena NT, Kim S, Lee MY, Jung JY, Choi JE. Photobiomodulation at a wavelength of $633 \mathrm{~nm}$ leads to faster functional recovery than $804 \mathrm{~nm}$ after facial nerve injury. J Biophotonics. In press 2021.

55. Wei L, Mousawi F, Li D, Roger S, Li J, Yang X, et al. Adenosine triphosphate release and $\mathrm{P} 2$ receptor signaling in Piezo1 channel-dependent mechanoregulation. Front Pharmacol 2019;10:1304.

56. Zhang Z, Shen Q, Wu X, Zhang D, Xing D. Activation of PKA/ SIRT1 signaling pathway by photobiomodulation therapy reduces A levels in Alzheimer's disease models. Aging Cell 2020;19:e13054.

57. Hu WP, Wang JJ, Yu CL, Lan CC, Chen GS, Yu HS. Heliumneon laser irradiation stimulates cell proliferation through photostimulatory effects in mitochondria. J Invest Dermatol 2007; 127:2048-57.
58. Heo JC, Park JA, Kim DK, Lee JH. Photobiomodulation (660 nm) therapy reduces oxidative stress and induces BDNF expression in the hippocampus. Sci Rep 2019;9:10114.

59. Li B, Wang X. Photobiomodulation enhances facial nerve regeneration via activation of PI3K/Akt signaling pathway-mediated antioxidant response. Lasers Med Sci. In press 2021.

60. Moccia F, Negri S, Shekha M, Faris P, Guerra G. Endothelial $\mathrm{Ca} 2+$ signaling, angiogenesis and vasculogenesis: just what it takes to make a blood vessel. Int J Mol Sci 2019;20:3962.

61. Goldsmith ZG, Dhanasekaran DN. G protein regulation of MAPK networks. Oncogene 2007;26:3122-42.

62. Zhang J, Wang X, Vikash V, Ye Q, Wu D, Liu Y, et al. ROS and ROS-mediated cellular signaling. Oxid Med Cell Longev 2016;2016:4350965.

63. Klimaschewski L, Hausott B, Angelov DN. The pros and cons of growth factors and cytokines in peripheral axon regeneration. Int Rev Neurobiol 2013;108:137-71.

64. Huang HT, Sun ZG, Liu HW, Ma JT, Hu M. ERK/MAPK and PI3K/ AKT signal channels simultaneously activated in nerve cell and axon after facial nerve injury. Saudi J Biol Sci 2017;24:1853-8.

65. Schmid RS, Pruitt WM, Maness PF. A MAP kinase-signaling pathway mediates neurite outgrowth on $L 1$ and requires Srcdependent endocytosis. J Neurosci 2000;20:4177-88.

66. Ghosh-Roy A, Wu Z, Goncharov A, Jin Y, Chisholm AD. Calcium and cyclic AMP promote axonal regeneration in Caenorhabditis elegans and require DLK-1 kinase. J Neurosci 2010;30:317583.

67. Rhee YH, Moon JH, Jung JY, Oh C, Ahn JC, Chung PS. Effect of photobiomodulation therapy on neuronal injuries by ouabain: the regulation of $\mathrm{Na}$, K-ATPase; Src; and mitogen-activated protein kinase signaling pathway. BMC Neurosci 2019;20:19.

How to cite this article: Choi JE. Proposed mechanisms of photobiomodulation (PBM) mediated via the stimulation of mitochondrial activity in peripheral nerve injuries. Med Lasers 2021;10:195-200.https://doi.org/10.25289/ML.2021.10.4.195 